\section{Proceso de alta hospitalaria, revisión de la literatura}

\author{
GONZALO EYMIN ${ }^{1,2, a}$, ANDRÉS AIZMAN², \\ MARCELO LOPETEGUI ${ }^{3,4}$, EFREN MANJARREZ ${ }^{1}$
}

\section{The discharge process}

Discharge is one of the most important processes that hospitalized patients must endure. This process is complex, requires coordination among several professionals and transfers an overwhelming amount of information to patients. Often, it is limited to the writing of the discharge summary, with a primary emphasis on the drug list. Since the rise of hospitalism in 1996, a greater emphasis has been placed on understanding this process and in developing interventions to make it more effective and safe. In our country, little is known about how this process is taking place. Probably the absence of financial penalties for readmissions has influenced in the lack of study and development of this process. In the USA the knowledge of the discharge process is well advanced, and several strategies have been developed for reducing adverse events, medication errors, and 30-days readmissions. Other interventions have increased patient satisfaction and the degree of knowledge about their conditions. The aim of this paper is to do a comprehensive review of the literature, to provide healthcare teams with various tools that could improve both the discharge process as well as the discharge summary. The final objective is to optimize the safety and satisfaction of our patients and the hospital metrics of quality.

(Rev Med Chile 2014; 142: 229-237)

Key words: Length of stay; Patient discharge; Patient readmission; Quality assurance, healthcare.

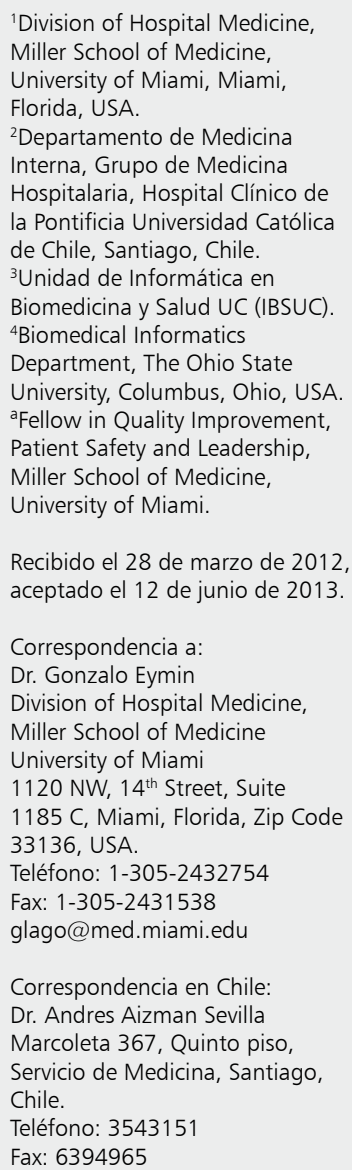

M uchos hospitales carecen de una política clara con respecto a las entregas de turno, los traslados entre servicios clínicos y el alta médica, procesos cruciales en la seguridad del paciente. En el alta hospitalaria se transfiere la responsabilidad entre el médico del hospital y el médico de atención primaria ${ }^{1}$, y una inadecuada planificación puede derivar en mayores reingresos, eventos adversos, y en insatisfacción del paciente $y$ familiares ${ }^{2-4}$. Casi la mitad de los pacientes hospitalizados experimenta errores, ya sea en el tratamiento, en exámenes, o en el seguimiento de resultados pendientes ${ }^{3}$, y entre $19 \%$ y $23 \%$ sufren algún evento adverso post-alta, siendo los más frecuentes los derivados de medicamentos ${ }^{5-7}$. A su vez, la tasa de reingresos a 30 días entre beneficiarios de Medicare (programa federal de Estados
Unidos de Norteamérica que paga ciertos gastos en salud en personas $\geq 65$ años) llega a $21 \%{ }^{8}$. En vista de esto, Joint Commission (JC) (Grupo independiente de Estados Unidos de Norteamérica sin fines de lucro que administra los programas de acreditación para hospitales y otros centros prestadores de cuidados de la salud) instruyó a las instituciones a realizar una conciliación de las indicaciones de medicamentos (CIM) acuciosa en cada paciente que experimenta un ingreso, un traslado dentro del hospital o un alta'. Medicare en Estados Unidos de Norteamérica penaliza económicamente a los hospitales con reingresos sobre cierto percentil a partir de $2012^{10}$. De igual modo, el pago asociado a diagnóstico en Estados Unidos de Norteamérica presiona a los hospitales a elaborar estrategias para reducir la duración de 
las hospitalizaciones y la readmisión a los 30 días.

En Chile es poco lo que se ha investigado en relación al proceso de alta. El objetivo del presente artículo es realizar una revisión no sistemática de la literatura de la situación actual del proceso de alta, y de las diversas estrategias destinadas a mejorarlo, de modo de proveer al lector con herramientas destinadas a optimizar tanto la seguridad de nuestros pacientes como los indicadores hospitalarios de calidad.

\section{Métodos}

Se realizó una revisión no sistemática bajo los términos mesh discharge planning, discharge summary, readmission, length of stay y quality assurance del buscador PubMed. Se seleccionaron estudios descriptivos y aleatorios que muestren la situación actual del proceso de alta así como estrategias destinadas a mejorarlo.

\section{Resultados}

El alta es un proceso complejo y multidisciplinario que debería ser especialmente minucioso en pacientes con alto riesgo de reingreso o de eventos adversos, entre los que se encuentran pacientes dados de alta a casas de reposo; con hospitalizaciones muy breves; con hospitalización en los últimos 6 meses; en cuidados paliativos; con pobre red social de apoyo; con baja comprensión en relación a cuidados médicos; con consumo de sustancias; con limitada disponibilidad de transporte a controles; con polifarmacia; usuarios de digoxina, aspirina y clopidogrel, insulina y warfarina; adultos mayores con depresión; con cáncer, insuficiencia cardiaca 0 accidente cerebro vascular ${ }^{11-15}$.

La epicrisis por su parte, a menudo carece de información relevante, tal como resultados de exámenes $(33 \%$ a $63 \%)$, evolución $(7 \%$ a $22 \%)$, medicamentos ( $2 \%$ a $40 \%)$, resultados pendientes $(65 \%)$, consejería ( $90 \%$ a $92 \%)$, y plan para controles $(2 \%$ a $43 \%)$, déficits también reportados en otros países ${ }^{16,17}$. La disponibilidad de la epicrisis en este estudio fue de $12 \%$ a $34 \%$ en el primer control, y de $51 \%-77 \%$ a las cuatro semanas ${ }^{16}$. Harding reportó que la falta de la epicrisis en el control dificultó la capacidad del médico de atención primaria para proporcionar una adecuada atención en $24 \%$ de los pacientes ${ }^{18}$. Callen mostró que entre $12,1 \%$ y $13,3 \%$ de las epicrisis redactadas a mano y en forma electrónica respectivamente contenía errores, siendo el más frecuente la omisión de medicamentos ${ }^{19}$. Were encontró que en sólo $25 \%$ de las epicrisis se mencionaba algún examen pendiente, y en sólo 13\% se mencionaban todos, encontrándose mención al control al alta en sólo $67 \%{ }^{20}$. Roy describió en su estudio que el médico de atención primaria no estaba en conocimiento de $62 \%$ de los resultados de exámenes pendientes al alta, considerándose que $37 \%$ de estos requerían una intervención ${ }^{21}$. Moore describió que sólo $36 \%$ de los pacientes realizaron los exámenes recomendados al alta, lo cual se correlacionó con un control más alejado, y con la ausencia de indicaciones por escrito ${ }^{22}$. Muchos médicos creen que debería existir un sistema de manejo de resultados pendientes que ayude al médico a recuperarlos y a hacerlos llegar a sus pacientes ${ }^{23}$. JC por otro lado, exige que la epicrisis contenga al menos: razón de la hospitalización, hallazgos significativos, procedimientos y tratamientos realizados, condición al alta, instrucciones, y la firma del tratante ${ }^{24}$. Los médicos de atención primaria opinan que debe al menos contener el diagnóstico, hallazgos anormales, resultados pendientes, controles, medicamentos, educación, y exámenes pendientes ${ }^{25-27}$. En cuanto al tipo de epicrisis, una encuesta reveló que los médicos de atención primaria prefieren una epicrisis estandarizada a una narrativa, ya que las primeras ofrecerían información más relevante $\mathrm{y}$ ordenada ${ }^{28}$. A continuación se desarrollan 10 ámbitos para los cuales existe evidencia que muestra beneficios para ciertas intervenciones y que incorporan todos los aspectos del proceso de alta. El análisis de estos ámbitos se inicia por la epicrisis, porque es el documento escrito que resume el plan del proceso del alta.

\section{Epicrisis}

Tradicionalmente la elaboración de la epicrisis se ha aprendido por imitación y de manera no estandarizada ${ }^{29}$. Myers demostró que un currículo estandarizado mejoró la calidad de las epicrisis realizadas por internos $(\mathrm{p}<0,05)$, mejoría que también se ha logrado en residentes de pediatría $(\mathrm{p}<0,002)^{30,31}$. Coit encontró que las epicrisis de residentes con menor carga laboral eran más completas y planificadas que las de residentes con carga laboral habitual $(\mathrm{p}<0,01)^{32}$. Varios estudios han logrado una mejoría en las epicrisis con el uso de planillas estandarizadas ${ }^{28,33,34}$. La Sociedad 
de Medicina Hospitalaria (SHM) desarrolló un punteo con los elementos necesarios para un alta ideal, focalizado principalmente en seguridad de medicamentos, educación de pacientes, y en los planes de seguimiento ${ }^{35}$. Los autores destacan que la epicrisis equivale a un "ingreso médico" al siguiente proveedor, y por lo tanto, merece tanta dedicación como un ingreso hospitalario. Sandler logró una reducción en el tiempo en que la epicrisis llegó al médico primario usando a los pacientes como mensajeros ${ }^{36}$.

\section{Conciliación de las indicaciones de medicamentos (CIM)}

En un estudio realizado por la Clínica Mayo, la falta de comunicación en entregas de turnos y durante el alta fue responsable de $50 \%$ de los errores de medicamentos y de $20 \%$ de los eventos $\operatorname{adversos}^{37}$. La CIM se realiza para evitar que se agreguen, modifiquen o supriman medicamentos inadvertidamente ${ }^{37}$, y requiere de una revisión exhaustiva de todos los medicamentos, de modo de mantener un listado exacto cada vez que cambia el tratamiento del paciente ${ }^{37,38}$. JC declaró la CIM como un objetivo nacional de seguridad de pacientes en un esfuerzo por minimizar los efectos adversos originados durante los cambios de responsables del tratamiento ${ }^{37}$. CIM se divide en verificación (registro de los medicamentos), aclaración (asegurar que la información es adecuada), y la conciliación (documentación de los cambios $^{39}$. La CIM debe realizarse al ingreso, en los cambios de servicios o de tratante, cuando cambia la condición clínica del paciente y al alta. Con el fin de tener una CIM segura, es crucial tener un registro inicial confiable. En este sentido Andersen demostró que una segunda entrevista de fármacos después del ingreso permite descubrir medicamentos no declarados en $69 \%$ de los pacientes, y que las fichas de médicos de atención primaria revelan fármacos no declarados en $70 \%$ de los $\operatorname{casos}^{40}$. Nester reportó que los registros de medicamentos obtenidos por farmacéuticos son más precisos que los obtenidos por enfermeras $(\mathrm{p}<0,001)^{41}$. En otro estudio, los farmacéuticos reconocieron errores en el $44 \%$ de los ingresos ${ }^{42}$. El farmacéutico clínico ha contribuido tanto a un mejor conocimiento y adherencia a los medicamentos por parte de los pacientes $(\mathrm{p}<0,01)$, como a una reducción de visitas no programadas al médico y de reingresos en adultos mayores con polifarmacia $(\mathrm{p}<0,05)^{43}$, beneficio no encontrado por Nazaret ${ }^{44}$. En un proyecto de mejoramiento de calidad, una segunda entrevista por un médico aumentó la exactitud de los registros tomados por enfermeras de $40 \%$ a $95 \%{ }^{45}$. Dada esta evidencia, el farmacéutico debería participar en la CIM al menos en los pacientes de alto riesgo ${ }^{46}$. En caso de no contar con farmacéuticos, el registro de medicamentos al ingreso debería ser realizado por lo menos por dos profesionales de la salud. Por último órdenes como "continuar o reiniciar todos los medicamentos" deberían ser evitadas. Ejemplos de planillas para CIM se pueden descargar desde la página de $\mathrm{SHM}^{47}$.

\section{Planificación del alta}

Varios estudios han demostrado que un equipo multidisciplinario permite mejorar la evaluación de las necesidades sociales de los pacientes y sus familiares al momento del alta ${ }^{4,48,49}$. Una revisión de The Cochrane Library mostró que un plan de alta estructurado adaptado a cada caso reduce discretamente las estadías hospitalarias $(-0,91$; IC de $95 \%=-1,55$ a $-0,27)$ y los reingresos en adultos mayores con patología médica (RR 0,85; IC de $95 \%=0,74-0,97)^{49}$. Mistiaen concluyó que las intervenciones que pueden tener un impacto positivo en la reducción de los problemas post alta en adultos, son aquéllas con componentes educativos, y las que combinan intervenciones pre y post alta ${ }^{50}$. Evans demostró que la planificación del alta desde el día 3 en pacientes de alto riesgo aumenta la probabilidad de regreso a casa ( $y$ no a una institución) $(\mathrm{p}=0,05)$, y disminuye el riesgo de reingreso a 30 días $(\mathrm{p}=0,001)^{51}$. Evans por su parte, demostró en una revisión sistemática que la planificación del alta en estos pacientes aumenta la posibilidad de permanecer en su hogar 6 a 12 meses después del alta (OR 1,4; IC del 95\% $=1,1-2,0)^{52}$. Una planificación integral del alta en adultos mayores también ha demostrado una reducción en los reingresos $(p=0,04)$, y en los costos $(p=0,02)^{53,54}$. El uso de una herramienta de 5 intervenciones (Hoja de ingreso geriátrica, envío de ésta al médico de atención primaria, planilla multidisciplinaria para identificar las barreras al alta, participación de farmacéutico, y controles post alta agendados) también ha reducido los reingresos $(\mathrm{OR}=0,59$; IC de $95 \%=0,34-0,97)$ y el uso del servicio de urgencias (OR $=0,25$; IC de $95 \%=0,10-0,62)$ a 30 días en población de edad avanzada ${ }^{55}$. 


\section{Seguimiento}

Medicare propone entregar a los pacientes instrucciones de alta claras y controles médicos tomados para reducir los reingresos. Si bien Grafft no encontró diferencias en reingresos entre los pacientes de medicina general con control post alta documentado y no documentado, Kyriacou encontró que los primeros tuvieron una mayor probabilidad de cumplir el plan de alta $(\mathrm{p}<0,001)^{56,57}$. Van Walvaren encontró que el control post alta con el médico del hospital puede reducir las muertes y los reingresos a los 30 días en comparación al médico de atención primaria (HR 0,95; IC de $95 \%=0,95-0,96)$ o al especialista (HR 0,97; IC de $95 \%=0,97-0,98)^{58}$. Hernández encontró que pacientes con insuficiencia cardiaca que tuvieron un control precoz tuvieron menos reingresos a los 30 días $(\mathrm{p}<0,001)^{59}$. Rytter encontró que las visitas domiciliarias post alta reducen el riesgo de reingreso $(p=0,03)$, y mejoran la adherencia a la medicación $(\mathrm{p}=0,05)$ en adultos mayores frági$\operatorname{les}^{60}$. Townsend encontró una menor frecuencia de reingresos múltiples con este tipo de intervención ${ }^{61}$. En otro estudio, este tipo de visitas mejoró la calidad de vida de los pacientes $(\mathrm{p}=0,02)$, la independencia $(\mathrm{p}=0,002)$ y redujo el número de días hospitalizados a 6 meses $(\mathrm{p}=0,01)^{62}$.

\section{Llamada telefónica}

Braun mostró que pacientes que reciben llamadas telefónicas a la semana, al mes y a los 3 meses del alta, están más satisfechos, realizan los exámenes solicitados con mayor frecuencia $(\mathrm{p}=0,02)$, y tienen una tendencia a menos reingresos $(\mathrm{p}=0,06)^{63}$. Harrison mostró que los pacientes contactados por teléfono dos semanas después del alta, tuvieron menos reingresos a 30 días $(p=0,04)^{64}$. Otro estudio encontró una tendencia hacia menos reingresos en pacientes con insuficiencia cardiaca llamados por estudiantes de enfermería ${ }^{65}$. Dudas demostró que llamados telefónicos por el farmacéutico dos días después del alta se asocian a una mayor satisfacción del paciente $(p=0,007)$, y a menos visitas a urgencias a 30 días $(\mathrm{p}=0,005)^{66}$. Schnipper demostró que el seguimiento telefónico, asociado a una CIM por un farmacéutico, y a la entrega de información al paciente se asoció a menos eventos adversos prevenibles a 30 días $(\mathrm{p}=0,01)^{67}$. No obstante, un meta-análisis concluyó que los estudios sobre llamadas telefónicas son de baja calidad, y que los resultados medidos son muy diferentes, no siendo posible una conclusión clara ${ }^{68}$.

\section{Educación}

Los médicos sobreestiman el tiempo invertido en educación y el grado de comprensión de los pacientes, lo cual resalta la necesidad de mejorar los métodos de comunicación al alta ${ }^{69}$. Una revisión de The Cochrane Library sugiere que la combinación de información verbal y escrita mejora el conocimiento y la satisfacción de los pacientes ${ }^{70}$. Koelling mostró que una charla de una hora por parte de una enfermera a pacientes con insuficiencia cardiaca, reduce el riesgo de re-hospitalización o de muerte $(\mathrm{p}=0,01)$ y los costos $(\mathrm{p}=0,03)^{71}$. Gattis demostró que los pacientes con insuficiencia cardiaca que recibieron educación y otras intervenciones por parte de un farmacéutico tuvieron una menor mortalidad por cualquier causa a 6 meses $(p=0,005)^{72}$. El uso de una cartilla con los medicamentos y su horario, ha demostrado mejorar la adherencia a los regímenes prescritos $(\mathrm{p}<0,001)^{73}$, beneficios también logrados con un programa de automedicación en que los pacientes son educados y se les entrega una mayor responsabilidad sobre sus medicamentos en el hospital $(\mathrm{p}<0,02)^{74}$. Otros métodos educativos exitosos son una entrevista estructurada realizada por residentes, las ayudas gráficas y la ilustración de horarios de administración ${ }^{75-77}$, y la técnica del teach-back, que consiste en pedirle al paciente que explique qué ha entendido de las instrucciones entregadas $^{78}$.

\section{Enfermera de apoyo de alta}

Una revisión sistemática muestra que la disponibilidad de una enfermera con conocimiento en gestión de casos impacta en la reducción de reingresos y de la estadía hospitalaria en personas mayores ${ }^{79}$, evidencia controvertida en pacientes con enfermedad pulmonar obstructiva crónica ${ }^{79-81}$. Stauffer mostró que un programa de transición para pacientes mayores con insuficiencia cardiaca manejado por enfermeras podría reducir los reingresos a 30 días (RR 0,48; IC de 95\% $=0,24-0,73)^{82}$.

\section{Evaluación geriátrica e intervenciones integrales después del alta}

Estas estrategias han demostrado reducir la duración de las estadías hospitalarias $(\mathrm{p}<0,05)$, el traslado a casas de reposo $(\mathrm{p}<0,05)$, mejorar 
la funcionalidad del paciente $(\mathrm{p}=0,04)$, y reducir los costos y la estadía hospitalaria de los reingresos $(\mathrm{p}=0,05)^{83}$. El beneficio en la duración de los reingresos también se encontró en el estudio de Naylor en pacientes mayores con problemas cardiacos graves $^{84}$.

\section{Entrenamiento}

Un modelo de "coaching" con enfermeras y trabajadores sociales en adultos mayores con insuficiencia cardiaca, consistente en rondas diarias para evaluar las necesidades y preparación de los pacientes para el alta, resultó en un aumento en la preparación para el auto cuidado $(\mathrm{p}=0,01)$, y en una disminución de la estadía hospitalaria en los reingresos ${ }^{85}$. El estudio "Care Transition Interventions" que consistió en promover la comunicación en los pacientes, mostró una reducción en los reingresos a 30 y 90 días $(p=0,04)$ y en los costos $(p=0,04)$ en pacientes con accidente cerebro vascular, insuficiencia cardiaca, enfermedad coronaria, arritmias cardiacas, enfermedad pulmonar obstructiva crónica, diabetes mellitus, estenosis espinal, fractura de cadera, enfermedad vascular periférica, trombosis venosa profunda y embolia pulmonar ${ }^{48,86}$.

\section{Apoyo de cuidados paliativos}

En Estados Unidos de Norteamérica 1/4 de los gastos de Medicare se desembolsan en el último año de vida ${ }^{87}$. El uso de servicios de cuidados paliativos en el hospital en pacientes con patología terminal, ha demostrado disminuir el número de reingresos a 6 meses $(p=0,025)^{88}$ y reducir el nivel de reingresos y de muertes de enfermos terminales en el hospital ${ }^{89}$. Gade también demostró que el uso de un equipo de cuidados paliativos disminuyó las admisiones a unidades de cuidados intensivos en los reingresos $(p=0,04)$, los costos $(p=0,001)$, y aumentó la estadía media en hospicios de pacientes con patología terminal $(\mathrm{p}=0,04)^{90}$.

\section{Intervenciones multidisciplinarias}

Un programa aplicado a pacientes mayores de alto riesgo, consistente en una evaluación por un farmacéutico, educación, una mayor planificación del alta y llamadas telefónicas, ha reducido los reingresos/uso del servicio de urgencias a 30 días $(\mathrm{p}=0,04)$, y ha prolongado el intervalo de tiempo entre el alta y el uso de servicios de salud $(\mathrm{p}=0,05)^{91}$. Otra intervención en pacientes con insuficiencia cardiaca consistente en una planificación del alta y un estrecho seguimiento ambulatorio, demostró ser útil en reducir los reingresos y muertes a 500 días $(p<0,001)$, y en prolongar el tiempo hasta la primera recaída, reingreso o muerte $(\mathrm{p}<0,001)^{92}$. El proyecto RED que consistió en un plan de alta desarrollado por una enfermera (que agenda los controles al alta, confirma la CIM, educa al paciente con folletos), y por un farmacéutico clínico (que llama por teléfono a los pacientes a los 2 y 4 días post alta), resultó en una reducción en la utilización de los hospitales $(\mathrm{p}=0,009)$, siendo más eficaz en pacientes con estadías hospitalarias dentro de los 6 meses previos al ingreso $(\mathrm{p}=0,01)^{93}$.

\section{Conclusión}

Las estrategias destinadas a mejorar el proceso de alta son múltiples. Algunas han sido estudiadas por separado mientras que otras como un paquete. La validez externa de muchas de estas intervenciones puede no ser aplicadas a nuestro medio, pero sientan las bases para desarrollar estudios en nuestro país. El sentido común dice que los requisitos mínimos de la epicrisis exigidos por JC deberían ser transversales y no discutibles (en virtud de la evidencia disponible). Frente a la ausencia de castigos económicos por estadías hospitalarias largas y reingresos, cada centro debería escoger un indicador que le permita evaluar el impacto de una determinada intervención, para lo cual resulta fundamental un adecuado registro de indicadores hospitalarios (tanto de procesos como de resultados). Más allá de los indicadores económicos que puedan verse beneficiados por estas medidas, cabe mencionar que la seguridad de los pacientes es impagable, y que si bien un porcentaje es una estadística, un solo paciente con un evento adverso puede ser una catástrofe para una institución.

\section{Referencias}

1. Manian FA. Whither continuity of care? N Engl J Med 1999; 340 (17): 1362-3.

2. Bull M, Hansen H, Gross C. Predictors of elder and family caregiver satisfaction with discharge planning. J Cardiovasc Nurs 2000; 14 (3): 76-87.

3. Moore C, Wisnivesky J, Williams S, McGinn T. Medical errors related to discontinuity of care from an inpatient 
to an outpatient setting. J Gen Intern Med 2003; 18 (8): 646-51.

4. Coleman E, Mahoney E, Parry C. Assessing the quality of preparation for posthospital care from the patient's perspective: the care transitions measure. Med Care 2005; 43 (3): 246-55.

5. Forster AJ, Clark H, Menard A, Dupuis N, Chernish $\mathrm{R}$, Chandok N, et al. Adverse events among medical patients after discharge from hospital. CMAJ 2004; 170 (3): 345-9.

6. Forster A, Murff H, Peterson J, Gandhi T, Bates D. Adverse drug events occurring following hospital discharge. J Gen Intern Med 2005; 20 (4): 317-23.

7. Forster A, Murff H, Peterson J, Gandhi T, Bates D. The incidence and severity of adverse events affecting patients after discharge from the hospital. Ann Intern Med 2003; 138 (3): 161-7.

8. Jencks S, Williams M, Coleman E. Rehospitalizations among patients in the Medicare fee-for-service program. N Engl J Med 2009; 360 (14): 1418-28.

9. Joint Commission. Joint Commission National Patient Safety Goals. [cited 2011 10/25]; Available from: http:// www.jointcommission.org/assets/1/18/National_Patient_Safety_Goals_6_3_111.PDF.

10. Stone J. Medicare Hospital Readmissions: Issues, Policy Options and PPACA. 2010 [cited 2011 11/25]; Available from: http://www.hospitalmedicine.org/AM/pdf/advocacy/CRS_Readmissions_Report.pdf.

11. Budnitz D, Shehab N, Kegler S, Richards C. Medication use leading to emergency department visits for adverse drug events in older adults. Ann Intern Med 2007; 147 (11): 755-65.

12. Dobrzanska L, Newell R. Readmissions: a primary care examination of reasons for readmission of older people and possible readmission risk factors. J Clin Nurs 2006; 15 (5): 599-606.

13. Strunin L, Stone M, Jack B. Understanding rehospitalization risk: can hospital discharge be modified to reduce recurrent hospitalization? J Hosp Med 2007; 2 (5): 297 304.

14. Boosting Care Transitions Resource Room Project Team. Risk Assessment Tool: the 8Ps. 2008 [cited 2011 1/25]; Available from: http://www.hospitalmedicine. org/ResourceRoomRedesign/RR_CareTransitions/ html_CC/06Boost/03_Assessment.cfm.

15. Arbaje A, Wolff J, Yu Q, Powe N, Anderson G, Boult C. Postdischarge environmental and socioeconomic factors and the likelihood of early hospital readmission among community-dwelling Medicare beneficiaries. Gerontologist 2008; 48 (4): 495-504.

16. Kripalani S, LeFevre F, Phillips C, Williams M, Basaviah
P, Baker D. Deficits in communication and information transfer between hospital-based and primary care physicians: implications for patient safety and continuity of care. JAMA, 2007. 297 (8): p. 831-41.

17. Rubak S, Mainz J. Communication between general practitioners and hospitals. Ugeskr Laeger 2000; 162 (5): 648-53.

18. Harding J. Study of discharge communications from hospital doctors to an inner London general practice. J R Coll Gen Pract 1987; 37 (304): 494-5.

19. Callen J, McIntosh J, Li J. Accuracy of medication documentation in hospital discharge summaries: A retrospective analysis of medication transcription errors in manual and electronic discharge summaries. Int J Med Inform 2010; 79 (1): 58-64.

20. Were M, Li X, Kesterson J, Cadwallader J, Asirwa C, Khan B, et al. Adequacy of hospital discharge summaries in documenting tests with pending results and outpatient follow-up providers. J Gen Intern Med 2009: 24 (9): 1002-6.

21. Roy C, Poon E, Karson A, Ladak-Merchant Z, Johnson $\mathrm{R}$, Maviglia $\mathrm{S}$, et al. Patient safety concerns arising from test results that return after hospital discharge. Ann Intern Med 2005; 143 (2): 121-8.

22. Moore C, McGinn T, Halm E. Tying up loose ends: discharging patients with unresolved medical issues. Arch Intern Med 2007: 167 (12): 1305-11.

23. Poon E, Gandhi T, Sequist T, Murff H, Karson A, Bates D. "I wish I had seen this test result earlier!": Dissatisfaction with test result management systems in primary care. Arch Intern Med 2004; 164 (20): 2223-8.

24. Kind A, Smith M. Documentation of Mandated Discharge Summary Components in Transitions from Acute to Subacute Care. [cited 2012 03/26] Available from: http://www.ahrq.gov/downloads/pub/advances2/ vol2/Advances-Kind_31.pdf

25. Pantilat S, Lindenauer P, Katz P, Wachter R. Primary care physician attitudes regarding communication with hospitalists. Am J Med 2001; 111 (9B): 15S-20S.

26. Newton J, Eccles M, Hutchinson A. Communication between general practitioners and consultants: what should their letters contain? BMJ 1992; 304 (6830): 8214.

27. Van Walraven C, Rokosh E. What is necessary for highquality discharge summaries? Am J Med Qual 1999; 14 (4): 160-9.

28. Van Walraven C, Duke S, Weinberg A, Wells P. Standardized or narrative discharge summaries. Which do family physicians prefer? Can Fam Physician 1998; 44: 62-9.

29. Boots R, Egerton W, McKeering H, Winter H. They just 
don't get enough! Variable intern experience in bedside procedural skills. Intern Med J 2009; 9 (4): 222-7.

30. Myers J, Jaipaul C, Kogan J, Krekun S, Bellini L, Shea J. Are discharge summaries teachable? The effects of a discharge summary curriculum on the quality of discharge summaries in an internal medicine residency program. Acad Med 2006; 81 (10 Suppl): S5-8.

31. Key-Solle M, Paulk E, Bradford K, Skinner A, Lewis M, Shomaker K. Improving the quality of discharge communication with an educational intervention. Pediatrics 2010; 126 (4): 734-9.

32. Coit M, Katz J, McMahon G. The effect of workload reduction on the quality of residents' discharge summaries. J Gen Intern Med 2011; 26 (1): 28-32.

33. Rao P, Andrei A, Fried A, González D, Shine D. Assessing quality and efficiency of discharge summaries. Am J Med Qual 2005; 20 (6): 337-43.

34. O’Leary K, Liebovitz D, Feinglass J, Liss D, Evans D, Kulkarni N, et al. Creating a better discharge summary: improvement in quality and timeliness using an electronic discharge summary. J Hosp Med 2009; 4 (4): 219-25.

35. Halasyamani L, Kripalani S, Coleman E, Schnipper J, van Walraven C, Nagamine J, et al. Transition of care for hospitalized elderly patients-development of a discharge checklist for hospitalists. J Hosp Med 2006; 1 (6): 354-60.

36. Sandler D, Mitchell J. Interim discharge summaries: how are they best delivered to general practitioners? Br Med J (Clin Res Ed) 1987; 295 (6612): 1523-5.

37. Greenwald JL, Halasyamani L, Greene J, LaCivita C, Stucky E, Benjamin B, et al. Making inpatient medication reconciliation patient centered, clinically relevant and implementable: a consensus statement on key principles and necessary first steps. J Hosp Med 2010; 5 (8): 477-85.

38. Cornish P, Knowles S, Marchesano R, Tam V, Shadowitz $\mathrm{S}$, Juurlink D, et al. Unintended medication discrepancies at the time of hospital admission. Arch Intern Med 2005; 165 (4): 424-9.

39. IHI. Reconcile medications at all transition points [cited 2011 7/12]; Available from: http://www.ihi.org/ knowledge/Pages/Changes/ReconcileMedicationsatAllTransitionPoints.aspx.

40. Andersen S, Pedersen A, Bach K. Medication history on internal medicine wards: assessment of extra information collected from second drug interviews and GP lists. Pharmacoepidemiol Drug Saf 2003; 12 (6): 491-8.

41. Nester T, Hale S. Effectiveness of a pharmacist-acquired medication history in promoting patient safety. Am J Health Syst Pharm 2002; 59 (22): 2221-5.

42. Gleason K, Groszek J, Sullivan C, Rooney D, Barnard C,
Noskin G. Reconciliation of discrepancies in medication histories and admission orders of newly hospitalized patients. Am J Health Syst Pharm 2004; 61 (16): 1689-95.

43. Al-Rashed S, Wright D, Roebuck N, Sunter W, Chrystyn $\mathrm{H}$. The value of inpatient pharmaceutical counselling to elderly patients prior to discharge. Br J Clin Pharmacol 2002; 54 (6): 657-64.

44. Nazareth I, Burton A, Shulman S, Smith P, Haines A, Timberal H. A pharmacy discharge plan for hospitalized elderly patients-a randomized controlled trial. Age Ageing 2001; 30 (1): 33-40.

45. Whittington J, Cohen H. OSF healthcare's journey in patient safety. Qual Manag Health Care 2004; 13 (1): 53-9.

46. Kripalani S, Jackson A, Schnipper J, Coleman E. Promoting effective transitions of care at hospital discharge: a review of key issues for hospitalists. J Hosp Med 2007; 2 (5): 314-23.

47. Boosting Care Transitions Resource Room Project Team. Boosting Care Transitions Resource Room/Medication raconciliation. [cited 2011 28/11] Available from: http://www.hospitalmedicine.org/ResourceRoomRedesign/RR_CareTransitions/html_CC/12ClinicalTools/05_ Medication.cfm.

48. Coleman E, Parry C, Chalmers S, Min S. The care transitions intervention: results of a randomized controlled trial. Arch Intern Med 2006; 166 (17): 1822-8.

49. Shepperd S, McClaran J, Phillips C, Lannin N, Clemson L, McCluskey A. Discharge planning from hospital to home. Cochrane Database Syst Rev 2010; (1): CD000313.

50. Mistiaen P, Francke A, Poot E. Interventions aimed at reducing problems in adult patients discharged from hospital to home: a systematic meta-review. BMC Health Serv Res 2007; 7: 47.

51. Evans R, Hendricks R. Evaluating hospital discharge planning: a randomized clinical trial. Med Care 1993; 31 (4): 358-70.

52. Hyde C, Robert I, Sinclair A. The effects of supporting discharge from hospital to home in older people. Age Ageing 2000; 29 (3): 271-9.

53. Naylor M. Comprehensive discharge planning for hospitalized elderly: a pilot study. Nurs Res 1990; 39 (3): 156-61.

54. Naylor M, Brooten D, Jones R, Lavizzo-Mourey R, Mezey M, Pauly M. Comprehensive discharge planning for the hospitalized elderly. A randomized clinical trial. Ann Intern Med 1994; 120 (12): 999-1006.

55. Dedhia P, Kravet S, Bulger J, Hinson T, Sridharan A, Kolodner K, et al. A quality improvement intervention to facilitate the transition of older adults from three 
hospitals back to their homes. J Am Geriatr Soc 2009; 57 (9): 1540-6.

56. Grafft C, McDonald F, Ruud K, Liesinger J, Johnson M, Naessens J. Effect of hospital follow-up appointment on clinical event outcomes and mortality. Arch Intern Med 2010; 170 (11): 955-60.

57. Kyriacou D, Handel D, Stein A, Nelson R. BRIEF REPORT: Factors affecting outpatient follow-up compliance of emergency department patients. J Gen Intern Med 2005; 20 (10): 938-42.

58. Van Walraven C, Mamdani M, Fang J, Austin PC. Continuity of care and patient outcomes after hospital discharge. J Gen Intern Med 2004; 19 (6): 624-31.

59. Hernández AF, Greiner MA, Fonarow GC, Hammill BG, Heidenreich PA, Yancy CW. Relationship between early physician follow-up and 30-day readmission among Medicare beneficiaries hospitalized for heart failure. JAMA 2010; 303 (17): 1716-22.

60. Rytter L, Jakobsen H, Rønholt F, Hammer A, Andreasen A, Nissen A, et al. Comprehensive discharge follow-up in patients' homes by GPs and district nurses of elderly patients. A randomized controlled trial. Scand J Prim Health Care 2010; 28 (3): 146-53.

61. Townsend J, Frank A, Piper M. Continuing rise in emergency admissions. Visiting elderly patients at home immediately after discharge reduces emergency readmissions. BMJ 1996; 313 (7052): 302.

62. Lim W, Lambert S, Gray L. Effectiveness of case management and post-acute services in older people after hospital discharge. Med J Aust 2003; 178 (6): 262-6.

63. Braun E, Baidusi A, Alroy G, Azzam Z. Telephone follow-up improves patients satisfaction following hospital discharge. Eur J Intern Med 2009; 20 (2): 221-5.

64. Harrison P, Hara P, Pope J, Young M, Rula E. The impact of postdischarge telephonic follow-up on hospital readmissions. Popul Health Manag 2011; 14 (1): 27-32.

65. Wheeler E, Waterhouse J. Telephone interventions by nursing students: improving outcomes for heart failure patients in the community. J Community Health Nurs 2006; 23 (3): 137-46.

66. Dudas V, Bookwalter T, Kerr K, Pantilat S. The impact of follow-up telephone calls to patients after hospitalization. Am J Med 2001; 111 (9B): 26S-30S.

67. Schnipper J, Kirwin J, Cotugno M, Wahlstrom S, Brown $\mathrm{B}$, Tarvin E, et al. Role of pharmacist counseling in preventing adverse drug events after hospitalization. Arch Intern Med 2006; 166 (5): 565-71.

68. Mistiaen P, Poot E. Telephone follow-up, initiated by a hospital-based health professional, for postdischarge problems in patients discharged from hospital to home. Cochrane Database Syst Rev 2006; (4): CD004510.
69. Calkins D, Davis R, Reiley P, Phillips R, Pineo K, Delbanco T, et al. Patient-physician communication at hospital discharge and patients' understanding of the postdischarge treatment plan. Arch Intern Med 1997; 157 (9): 1026-30.

70. Johnson A, Sandford J, Tyndall J. Written and verbal information versus verbal information only for patients being discharged from acute hospital settings to home. Cochrane Database Syst Rev 2003; (4): CD003716.

71. Koelling T, Johnson M, Cody R, Aaronson K. Discharge education improves clinical outcomes in patients with chronic heart failure. Circulation 2005; 111 (2): 179-85.

72. Gattis W, Hasselblad V, Whellan D, O'Connor C. Reduction in heart failure events by the addition of a clinical pharmacist to the heart failure management team: results of the Pharmacist in Heart Failure Assessment Recommendation and Monitoring (PHARM) Study. Arch Intern Med 1999; 159 (16): 1939-45.

73. Raynor D, Booth T, Blenkinsopp A. Effects of computer generated reminder charts on patients' compliance with drug regimens. BMJ 1993; 306 (6886): 1158-61.

74. Lowe C, Raynor D, Courtney E, Purvis J, Teale C. Effects of self medication programme on knowledge of drugs and compliance with treatment in elderly patients. BMJ 1995; 310 (6989): 1229-31.

75. Louis-Simonet M, Kossovsky M, Sarasin F, Chopard P, Gabriel V, Perneger T, et al. Effects of a structured patient-centered discharge interview on patients' knowledge about their medications. Am J Med 2004; 117 (8): 563-8.

76. Katz M, Kripalani S, Weiss B. Use of pictorial aids in medication instructions: a review of the literature. Am J Health Syst Pharm 2006; 63 (23): 2391-7.

77. Kripalani S, Robertson R, Love-Ghaffari M, Henderson L, Praska J, Strawder A, et al. Development of an illustrated medication schedule as a low-literacy patient education tool. Patient Educ Couns 2007; 66 (3): 36877.

78. Schillinger D, Piette J, Grumbach K, Wang F, Wilson C, Daher C, et al. Closing the loop: physician communication with diabetic patients who have low health literacy. Arch Intern Med 2003; 163 (1): 83-90.

79. Chiu W, Newcomer R. A systematic review of nurseassisted case management to improve hospital discharge transition outcomes for the elderly. Prof Case Manag 2007; 12 (6): 330-6; quiz 337-8.

80. Nissen I, Jensen M. Nurse-supported discharge of patients with exacerbation of chronic obstructive pulmonary disease. Ugeskr Laeger 2007; 169 (23): 2220-3.

81. Kwok T, Lum C, Chan H, Ma H, Lee D, Woo J. A randomized, controlled trial of an intensive community 
Alta hospitalaria. Revisión - G. Eymin et al

nurse-supported discharge program in preventing hospital readmissions of older patients with chronic lung disease. J Am Geriatr Soc 2004; 52 (8): 1240-6.

82. Stauffer B, Fullerton C, Fleming N, Ogola G, Herrin J, Stafford $\mathrm{P}$, et al. Effectiveness and cost of a transitional care program for heart failure: a prospective study with concurrent controls. Arch Intern Med 2011; 171 (14): 1238-43.

83. Nikolaus T, Specht-Leible N, Bach M, Oster P, Schlierf G. A randomized trial of comprehensive geriatric assessment and home intervention in the care of hospitalized patients. Age Ageing 1999; 28 (6): 543-50.

84. Naylor M, McCauley K. The effects of a discharge planning and home follow-up intervention on elders hospitalized with common medical and surgical cardiac conditions. J Cardiovasc Nurs 1999; 14 (1): 44-54.

85. Bull M, Hansen H, Gross C. A professional-patient partnership model of discharge planning with elders hospitalized with heart failure. Appl Nurs Res 2000; 13 (1): 19-28.

86. Voss R, Gardner R, Baier R, Butterfield K, Lehrman S, Gravenstein $S$. The care transitions intervention: translating from efficacy to effectiveness. Arch Intern Med 2011; 171 (14): 1232-7.

87. Hogan C, Lunney J, Gabel J, Lynn J. Medicare beneficiaries' costs of care in the last year of life. Health Aff
(Millwood) 2001; 20 (4): 188-95.

88. Nelson C, Chand P, Sortais J, Oloimooja J, Rembert G. Inpatient palliative care consults and the probability of hospital readmission. Perm J 2011; 15 (2): 48-51.

89. Fromme E, Bascom P, Smith M, Tolle S, Hanson L, Hickam D, et al. Survival, mortality, and location of death for patients seen by a hospital-based palliative care team. J Palliat Med 2006; 9 (4): 903-11.

90. Gade $G$, et al. Impact of an inpatient palliative care team: a randomized control trial. J Palliat Med, 2008. 11 (2): p. 180-90.

91. Koehler B, Richter K, Youngblood L, Cohen B, Prengler I, Cheng D, et al. Reduction of 30-day postdischarge hospital readmission or emergency department (ED) visit rates in high-risk elderly medical patients through delivery of a targeted care bundle. J Hosp Med 2009; 4 (4): 211-8.

92. Atienza F, Anguita M, Martínez-Alzamora N, Osca J, Ojeda S, Almenar L, et al. Multicenter randomized trial of a comprehensive hospital discharge and outpatient heart failure management program. Eur J Heart Fail 2004; 6 (5): 643-52.

93. Jack B, Chetty V, Anthony D, Greenwald J, Sánchez $\mathrm{G}$, Johnson A, et al. A reengineered hospital discharge program to decrease rehospitalization: a randomized trial. Ann Intern Med 2009; 150 (3): 178-87. 